\title{
Cosmogenic neutrinos as a probe of the transition from Galactic to extragalactic cosmic rays
}

\author{
Hajime Takami $^{\text {a }}{ }^{1}$, Kohta Murase ${ }^{\mathrm{b}}$, Shigehiro Nagataki ${ }^{\mathrm{b}}$, and \\ Katsuhiko Sato ${ }^{\text {a,c,d }}$ \\ ${ }^{a}$ Department of Physics, School of Science, the University of Tokyo, 7-3-1 Hongo, \\ Bunkyo-ku, Tokyo 113-0033, Japan \\ ${ }^{\mathrm{b}}$ Yukawa Institute for Theoretical Physics, Kyoto University, Oiwake-cho, \\ Kitashirakawa, Sakyo-ku, Kyoto, 606-8502, Japan \\ ${ }^{\mathrm{c}}$ Research Center for the Early Universe, School of Science, the University of \\ Tokyo, 7-3-1 Hongo, Bunkyo-ku, Tokyo 113-0033, Japan \\ ${ }^{\mathrm{d}}$ Institute of Physics and Mathematics for Universe, the University of Tokyo, \\ Kashiwa, Chiba, 277-8582, Japan
}

\begin{abstract}
There are two promising scenarios that explain the ankle, which is a dip in the spectrum of cosmic rays at $\sim 10^{19} \mathrm{eV}$. A scenario interprets the ankle as the transition from Galactic to extragalactic cosmic rays (ankle-transition scenario), while the other is that the dip caused by pair production on the cosmic microwave background radiation (proton-dip scenario). In this paper, we consider whether cosmogenic neutrinos can be a clue to judge which scenario is favored. We calculated the fluxes of cosmogenic neutrinos following these scenarios with plausible physical parameter sets, and found several important features as follows. First of all, the neutrino flux at $\sim 10^{20} \mathrm{eV}$ becomes much higher in the ankle-transition scenario as long as the maximum energy of the cosmic rays at sources is sufficiently high. On the other hand, the neutrino spectrum has a characteristic peak at $\sim 10^{16} \mathrm{eV}$ in the protondip scenario on the condition that extragalactic protons significantly contribute to the observed cosmic rays down to $10^{17} \mathrm{eV}$. Thus, we conclude cosmogenic neutrinos should give us a clue to judge which scenario is favored, unless these features are masked by the neutrino background coming from possible, powerful neutrino sources such as AGNs and GRBs. We also found an interesting feature that the neutrino flux at $\sim 10^{18} \mathrm{eV}$ depends only on the cosmological evolution of the cosmic ray sources. That means cosmogenic neutrinos with the energy bring us information on

1 E-mail addresses: takami@utap.phys.s.u-tokyo.ac.jp (H.Takami), kmurase@yukawa.kyoto-u.ac.jp（K.Murase), nagataki@yukawa.kyoto-u.ac.jp (S.Nagataki), sato@phys.s.u-tokyo.ac.jp (K.Sato)
\end{abstract}


the cosmological evolution of the sources of ultra-high energy cosmic rays. Finally, we compare the fluxes of cosmogenic neutrinos with the expected sensitivity curves of several neutrino detectors, and conclude the detection of cosmogenic neutrinos in the near future is promising. 
Key words: Ultra-high-energy cosmic rays; Ultra-high-energy neutrinos

\section{Introduction}

What energy is the transition point of Galactic cosmic rays (GCRs) and extragalactic cosmic rays (EGCRs) in cosmic ray spectrum is an intriguing problem in cosmic ray astrophysics. The observed spectrum of cosmic rays, over more than 12 orders of magnitude in energy, can be described by a power-law shape with several spectral breaks [1]. The spectral breaks are interpreted as the transition points of sources to mainly contribute to the observed flux of cosmic rays. A spectral break at $\sim 10^{15.5} \mathrm{eV}$, so-called knee, has been thought of as the appearance of a maximum acceleration energy of protons at energetic objects in our Galaxy, like supernova remnants.

At $\sim 10^{19} \mathrm{eV}$, a spectral dip, called ankle, has been observed. Traditionally, the ankle has been interpreted as a transition point from GCRs with a steep spectrum $\left(\propto E^{-3.1}\right)$ to EGCRs with a harder spectrum $\left(\propto E^{-2.0 \sim-2.3}\right)$. This is partly because cosmic rays above $10^{19} \mathrm{eV}$ could not be confined in the Galaxy by the Galactic magnetic field and their arrival distribution is highly isotropic. We call this traditional scenario ankle-transition scenario throughout this paper. In this scenario, it is an open problem how GCRs are accelerated up to $10^{19} \mathrm{eV}$ in our Galaxy. On the other hand, it has been suggested recently that extragalactic protons with a steep injection spectrum $\left(\propto E^{-2.6 \sim-2.7}\right)$ can reproduce the ankle as a spectral dip due to Bethe-Heitler pair-creation with cosmic microwave background (CMB) photons $[2,3]$. This scenario, called proton-dip scenario throughout this paper, requires extragalactic protons at least down to $10^{18} \mathrm{eV}$. In the proton-dip scenario, the second knee, which is another spectral break at $\sim 10^{17.7} \mathrm{eV}$, is interpreted as a transition point from GCRs to EGCRs. It is an intriguing problem for the determination of cosmic ray origin which scenario is favored.

It is difficult to distinguish the two scenarios based on the energy spectrum of cosmic rays in observations. One of the key clues to determine the transition point is cosmic ray composition. In the ankle-transition scenario, the composition of GCRs at $\sim 10^{19} \mathrm{eV}$ is expected to be dominated by heavy nuclei because of their capability of accelerating up to such high energy, while proton-dominated composition is required in the proton-dip scenario. Thus, composition measurements may provide us with useful information on the transition scenarios. However, the results of composition measurements are difficult to constrain transition scenarios because the uncertainty of hadronic interaction models in the simulations of extensive air showers obstructs the accurate determination of the composition $[4,5]$. 
In this study, we focus on another key element, cosmogenic neutrinos, which are generated by photopion production between ultra-high-energy cosmic rays (UHECRs) propagating in intergalactic space and cosmic background photons, and through the successive decay of producing pions and muons. Cosmogenic neutrinos are a definite probe of EGCRs. The estimations of the flux of cosmogenic neutrinos have been performed since the discovery of the CMB $[6,7,8,9,10]$. A recent work of Ref. [11] pointed out that cosmogenic neutrinos, coupled with UHECR results, would provide a sufficient description of the properties of UHECR sources.

Cosmic background radiation with the energy higher than the CMB (e.g., infrared (IR), optical, ultraviolet (UV)) can significantly contribute to the total flux of cosmogenic neutrinos because there is a large number of UHECRs which can generate neutrinos by interactions with such higher energy photons though the number of such photons is much smaller than the CMB $[12,13,14]$. Recent progress in the observations of high-redshift Universe allows constructing detailed models of the spectral energy distribution (SED) of IR to UV background (IR/UV below) radiation [15,16]. Refs. [13,14] discussed the neutrino flux using the SED model constructed by Ref. [16]. Ref. [13] considered the propagation of UHE protons and estimated the resultant flux of cosmogenic neutrinos. Based on this work, it was suggested in Ref. [17] that cosmogenic neutrinos might be a key clue for determining the transition energy, but detailed discussions were not performed. On the other hand, the authors of Ref.[14] estimated the fluxes of cosmogenic neutrinos on the assumptions of both a pure proton and mixed composition models.

Motivated by these studies, we discuss whether cosmogenic neutrinos can be a clue to judge which transition scenario is favored in detail in this study. We calculate the spectra of cosmogenic neutrinos following the two transition scenarios for this purpose for plausible physical parameter sets. Since the neutrino fluxes depend on several physical parameters, like the maximum acceleration energy of UHECRs, the minimum energy of EGCRs, the spectral shape of UHECRs, and the cosmological evolution of UHECR sources, we also investigate the parameter dependence of the neutrino fluxes and the capability of distinguishing between the two transition scenarios. We adopt an IR/UV background model other than a model used in Refs. $[12,13,14]$. The neutrino fluxes are normalized by the fluxes and spectral shapes of accompanying UHECRs obtained in observations. For simplicity, extragalactic magnetic field is neglected.

The composition of EGCRs are assumed to be purely protons in this study. EGCR composition is an interesting problem in itself. The High Resolution Fly's Eye (HiRes) reported that the composition of cosmic rays above $10^{18} \mathrm{eV}$ is dominated by protons as a result of $\left\langle X_{\max }\right\rangle$, the averaged depth of the shower maximum, measurement [4]. A recent result by Pierre Auger Observa- 
tory (PAO) is consistent with the HiRes result within systematic uncertainty [5]. On the other hand, studies of muon content in the extensive air shower, another observable for UHECR composition, indicate a significant fraction of heavy nuclei above $10^{19} \mathrm{eV}[18,19]$. The accurate interpretation of these composition measurements is difficult because of our poor knowledge of hadronic interactions at ultra-high-energy, as mentioned above. The PAO also reports the positional correlation between the arrival directions of the highest energy cosmic rays above $5.7 \times 10^{19} \mathrm{eV}$ and nearby active galactic nuclei (AGNs) $[20,21]$. If these AGNs are really the sources of the observed events, this fact implies the highest energy cosmic rays are dominated by protons because of small deflections by the Galactic magnetic field. This implication is independent of the composition measurements. Thus, an assumption that EGCRs are dominated by protons up to the highest energies is reasonable.

This paper is organized as follows: in Section 2, we explain our calculation method of cosmogenic neutrino fluxes in detail. In Section 3, we address our results and discuss the detectability of cosmogenic neutrinos taking neutrino oscillation into account. In Section 4, several uncertainties on the neutrino flux are discussed. We conclude in Section 5.

\section{Our Calculation Method}

In this section, our calculation method of the flux of cosmogenic neutrinos is explained. The neutrino flux can be obtained by calculating the number of neutrinos produced by propagating protons injected from a source, and then by integrating such neutrinos over all sources. We explain the propagation of UHE protons and their interactions with cosmic background photons in Section 2.1. In Section 2.2, our treatment of neutrino production is described. Then, we represent UHECR source models to calculate the total neutrino flux in Section 2.3.

\subsection{Propagation of UHE Protons}

Protons propagating in intergalactic space interact with cosmic background photons and lose their energies through particle productions [9,22]. They also lose their energies adiabatically due to the cosmic expansion since they propagate over cosmological distance. We consider two interactions with cosmic background photons: photopion production and Bethe-Heitler pair creation, and adiabatic energy-loss.

For cosmic background photons, we take into account not only the CMB 
photons but also IR/UV background photons. Background photons with the energies higher than the CMB photons allow protons with the energies lower than the threshold of photopion production with the CMB to generate neutrinos. Thus, they increase the neutrino flux significantly while they do not change UHECR spectrum because of the smaller number density of IR/UV photons compared with the CMB [13]. We adopt the best-fit model of the SED of IR/UV background photons calculated in Ref.[15].

Photopion production is the most essential process in this study. This process is treated stochastically. The mean free path of photopion production for a proton with energy $E$, in an isotropic photon field, $\lambda(E, z)$, is calculated as $[23]$

$$
\frac{1}{\lambda(E, z)}=\frac{1}{8 \beta E^{2}} \int_{\epsilon_{\mathrm{th}}}^{\infty} d \epsilon \frac{1}{\epsilon^{2}} \frac{d n_{\gamma}}{d \epsilon}(\epsilon, z) \int_{s_{\min }}^{s_{\max }} d s \sigma(s)\left(s-m_{p}{ }^{2} c^{4}\right)
$$

where $s_{\min }=\left(m_{\pi^{0}}+m_{p}\right)^{2} c^{4}, s_{\max }=m_{p}{ }^{2} c^{4}+2 E \epsilon(1+\beta), \epsilon_{\mathrm{th}}=\left(s_{\min }-\right.$ $\left.m_{p}{ }^{2} c^{4}\right)[2 E(1+\beta)]^{-1} \cdot m_{p}, m_{\pi_{0}}, c, \beta, \epsilon$, and $s$ are the proton mass, the neutral pion mass, the speed of light, the velocity of the proton in the unit of the speed of light, the energy of cosmic background photons, and the Lorentz invariant energy squared, respectively. $\sigma(s)$ is the total cross section of photopion production and $d n_{\gamma} / d \epsilon$ is the differential number density of cosmic background photons. $\sigma(s)$ is calculated by GEANT4, a Monte-Carlo simulation tool-kit which can simulate photomeson productions [24]. The GEANT4 can well reproduce the experimental total cross section of photopion production.

The mean free paths in intergalactic space are calculated and used for every $\Delta z=0.1$ up to $z=5$. The mean free paths at several redshifts $z$ are shown in Fig.1. The mean free path at $z=0$ rapidly decreases above $8 \times 10^{19} \mathrm{eV}$ where the channel of interactions with the CMB opens, which makes sharply cosmic ray spectrum steepening, so-called Greisen-Zatsepin-Kuz'min (GZK) steepening $[25,26]$. At $z=0$, the minimum length of the mean free path $\lambda_{\min }(z)$ is about $4 \mathrm{Mpc}$. The interaction points of propagating protons are determined by a method used in Ref.[27], based on $\lambda(E, z)$ and $\lambda_{\min }(z)$.

The inelasticity of photopion production, $K(s)$, is approximated by a conventional method as $K(s)=\left[1-\left(m_{\mathrm{CR}}^{2}-m_{\pi}^{2}\right) / s\right] / 2$ for single pion production, where $m_{\mathrm{CR}}$ and $m_{\pi}$ are the mass of the cosmic ray after an interaction (proton or neutron), the pion mass generated in the reaction, respectively. For multipion production (see Section 2.2), we approximately adopt this formula by replacing $m_{\pi}$ with the total mass of pions. This assumption does not almost affect the flux of cosmogenic neutrinos shown in Section 3. A typical inelasticity is 0.23 at $s \sim 1.6 \mathrm{GeV}^{2}$, which is the Lorentz invariant energy squared at the delta resonance in the total cross section of photopion production. 


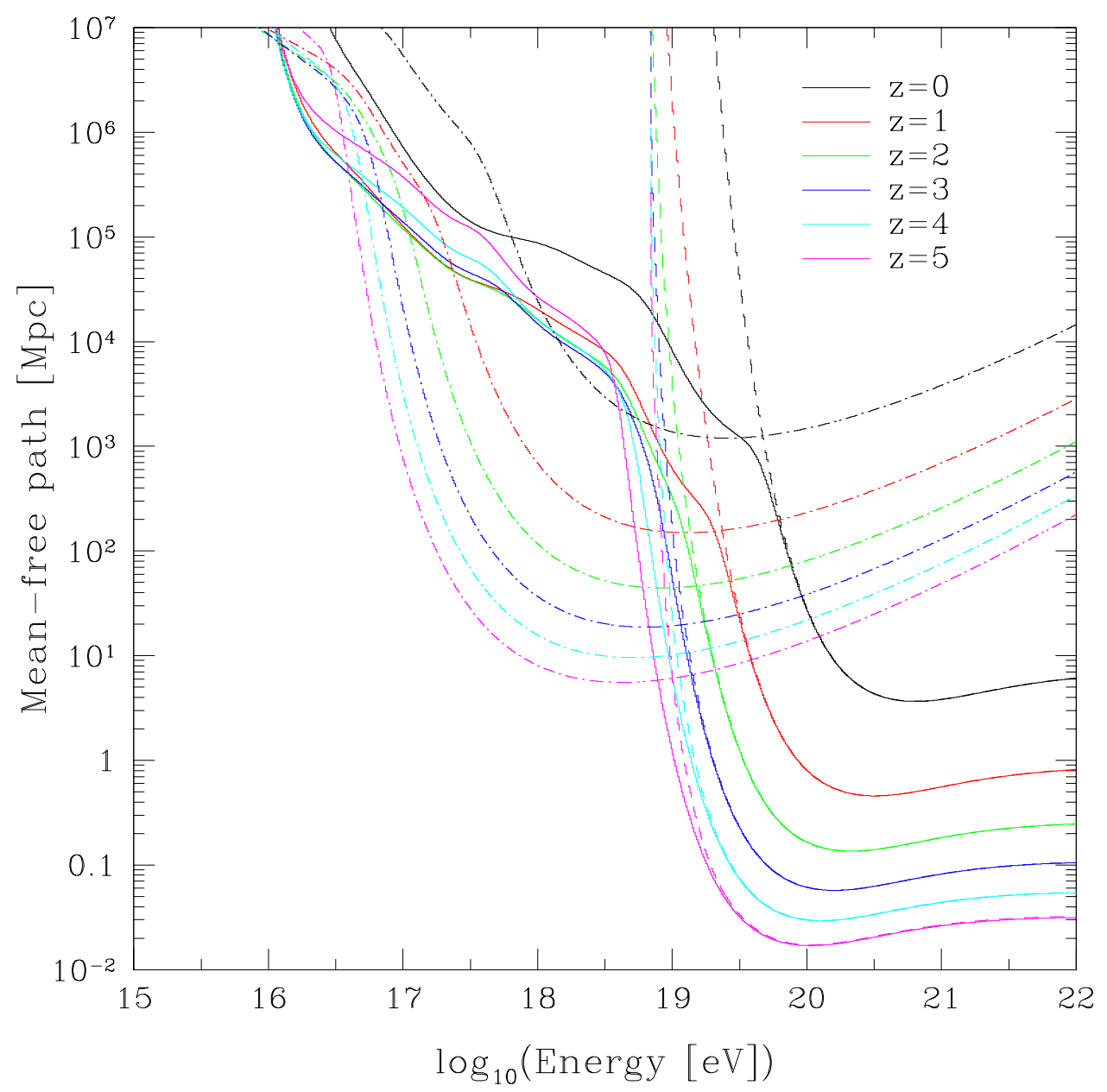

Fig. 1. Mean free paths of protons for photopion production in cosmic background radiation including IR/UV photons (solid lines) and only CMB photons (dashed lines) at six redshifts. The energy-loss lengths of Bethe-Heitler pair creation of protons in cosmic background radiation from the CMB to IR/UV are also shown (dot-dashed lines).

Cosmic background photons also make protons lose their energies through Bethe-Heitler pair creation, $p \gamma \longrightarrow p e^{+} e^{-}$. Fig.1 also shows the energy-loss lengths of this process which are calculated following an analytical fitting function given by Ref.[28]. This energy-loss process can be treated in a continuous energy-loss approximation since its inelasticity is very small $\left(2 m_{e} / m_{p} \sim 10^{-3}\right)$. Since the energy-loss length is much shorter than the interaction length of photopion production for IR/UV photons at high-redshift Universe, neutrino production related to IR/UV photons mainly occurs at about less than $\mathrm{z}=1$.

The energy-loss rate of protons with energy $E$ due to the cosmic expansion is 
expressed as

$$
\frac{d E}{d t}=-\frac{\dot{a}}{a} E=-H_{0}\left[\Omega_{m}(1+z)^{3}+\Omega_{\Lambda}\right]^{1 / 2} E
$$

where $\Lambda$ CDM cosmology with $\Omega_{m}=0.3, \Omega_{\Lambda}=0.7$, and $H_{0}=71 \mathrm{~km} \mathrm{~s}^{-1} \mathrm{Mpc}^{-1}$ is assumed. We also treat this process as continuous process during propagation.

The spectra of UHE protons and cosmogenic neutrinos are calculated as follows: first, we consider protons with $10^{16}-10^{22} \mathrm{eV}$ ejected from a source. This energy range is divided into 60 bins with $\Delta\left(\log _{10} E\right)=0.1$ and 2000 protons are ejected in each bin initially regardless of UHECR injection spectrum. The propagation of protons is calculated taking the energy-loss processes and pion production into account, and then the resultant spectra of UHE protons and neutrinos at the Earth are recorded for each initial energy bin. The calculation of propagation stops when the energies of propagating protons reach $10^{15} \mathrm{eV}$. Neutrinos are assumed to lose their energies only due to the adiabatic energy loss. Then, the resultant spectra sum up over all initial energy bins weighted by a given injection spectrum to obtain the spectra of UHE protons and cosmogenic neutrinos from a source. Finally, the total spectra of protons and cosmogenic neutrinos are calculated by integrating their spectra from a source over all sources taking UHECR source-evolution models into account. The injection spectra and UHECR source-evolution models adopted in this study are explained in Section 2.3.

\subsection{Neutrino Production}

Cosmogenic neutrinos are produced by the decay of charged pions generated by photopion production of propagating protons with cosmic background photons. A charged pion decays into a muon and muon neutrino, $\pi^{+} \rightarrow \mu^{+} \nu_{\mu}$ or $\pi^{-} \rightarrow \mu^{-} \overline{\nu_{\mu}}$. The muon decays into an electron and neutrinos, $\mu^{-} \rightarrow e^{-} \overline{\nu_{e}} \nu_{\mu}$ or $\mu^{+} \rightarrow e^{+} \nu_{e} \overline{\nu_{\mu}}$.

There are many reaction modes for pions in photopion production, since protons with sufficient energies could produce more than one pions. The GEANT4, which is used to calculate the total cross section, can also calculate exclusive cross section of each reaction mode, but it has some problems in parametrization. Therefore, we combine the experimental data of exclusive cross sections similarly to Ref.[29]. In addition, it takes a large CPU time to fully simulate photopion production one by one. Hence, we shall take a simpler treatment to calculate neutrino generation, as explained below.

We consider $p \gamma \rightarrow p \pi^{0}, n \pi^{+}$as single pion production processes, and $p \gamma \rightarrow$ 
$p \pi^{+} \pi^{-}, n \pi^{0} \pi^{+}, p \pi^{0} \pi^{0}$ as double pion production processes. We adopt experimental results of the cross sections of these reactions below $s \sim 3 \mathrm{GeV}^{2}$ like Refs. $[29,30,31]$. These cross sections are not confirmed well by experiments at $s>3 \mathrm{GeV}^{2}$. Therefore, we simply extrapolate the cross sections from $s \sim 3 \mathrm{GeV}^{2}$ to higher energy. Then, we regard the total cross section minus the contributions of single and double pion production as the cross section of triple pion production, which generates $\pi^{0}, \pi^{+}$and $\pi^{-}$. The above treatment is sufficient unless multi-pion production processes are significant.

Once we judge that a propagating proton raises photopion production in calculation, the counterpart photon energy $\epsilon$, and $s$ are determined by a method used in Ref.[23]. Next, it is determined which reaction is realized based on the probabilities proportional to the cross section of each reaction mode at $s$. The total energy of the produced pions is given by $K(s) E . K(s) E$ is the energy of the pion for single pion production. For double or triple pion production, we assume the total energy, $K(s) E$, to be divided equally into all pions. Generated charged pions decay into muons and muon neutrinos. The energies of the products are calculated using the two body decay algorithm. The energies of the products of muon decay are calculated using the three body decay algorithm. The pions and muons decay immediately.

Neutrinos are also produced by the beta decay of neutrons, $n \rightarrow p e^{-} \overline{\nu_{e}}$, which result from charged pion production. These neutrinos can contribute to the observed neutrino flux if the mean free path of the beta decay, $\gamma c \tau=$ $0.92\left(E / 10^{20} \mathrm{eV}\right) \mathrm{Mpc}$, is shorter than that of photopion production of a neutron which is assumed to be equal to that of a proton. At $z=0$, for example, the mean free path of the photopion production is comparable with that of beta decay of a neutron with $10^{20.6} \mathrm{eV}$. Hence, the neutron beta decay is also an important process for neutrino productions. This process generates only anti-electron neutrinos. The energies of the products are calculated using the three body decay algorithm.

\subsection{UHECR Source Models}

The total flux of cosmogenic neutrinos is calculated by integrating a neutrino flux from a source over all sources. The cosmological evolution of the number density and luminosity density of UHECR sources strongly affects on the flux of cosmogenic neutrinos. In this study, we consider four source-evolution models in which sources are uniformly distributed with cosmological evolution up to $z=5$.

The first is a uniform distribution without cosmological source-evolution (UNF). The second is a model following the luminosity density evolution of quasars 
(QSO), which is parametrized by Ref. [32]

$$
f_{\mathrm{QSO}}(z) \propto\left\{\begin{array}{cc}
(1+z)^{3} & (z<1.3) \\
\text { Constant } & (1.3<z<2.7) \\
\exp \left(1-\frac{z}{2.7}\right) & (2.7<z)
\end{array}\right.
$$

The third follows a star formation rate (SFR) deduced from the reconstruction of a IR/UV background flux used in this study,

$$
f_{\mathrm{SFR}}(z) \propto\left\{\begin{array}{cc}
(1+z)^{3.5} & (z<1.2) \\
(1+z)^{-1.2} & (1.2<z)
\end{array} .\right.
$$

The fourth, the last model is motivated by an assumption that the observed UHECRs come from gamma-ray bursts (GRBs) [33]. Although the redshift distribution of GRBs is still controversial and it is different between pre-Swift and Swift bursts (e.g., [34]), one plausible rate history is that the GRB rate is more enhanced at higher redshifts due to metalicity effect [35]. It infers that the progenitors of GRBs would favor metal-poor stars, and host galaxies of GRBs would tend to have low metalicities [36,37]. In this paper, we shall adopt such an evolution model, and refer this GRB rate model as a "GRB-metalicity anticorrelation" model. More specifically, we shall assume a following rate history, $f_{\mathrm{GRB}}(z) \propto(1+z)^{1.4} f_{\mathrm{SFR}}(z)$, which is used in Ref.[38] based on the calculation of Ref.[39]. We use this parametrization in our fourth source-evolution model (SFR+GRBMAC below). As for the star formation rate, $f_{\mathrm{SFR}}(z)$ in the third model is adopted. Our results do not depend on the local GRB rate which is somewhat uncertain so far, because we use the observed UHECR flux for the normalization of the flux of cosmogenic neutrinos. However, note that the lower local rate requires higher baryon loading per GRB if UHECRs come from GRBs.

As for the proton injection spectra at all sources, we assume a simple powerlaw spectrum,

$$
\frac{d N}{d E} \propto E^{-\alpha} \Theta\left(E_{\max }-E\right) \Theta\left(E-E_{\min }\right),
$$

where $\alpha, E_{\max }$ and $E_{\min }$ are a spectral index, the maximum acceleration energy of protons and the minimum energy of protons at sources respectively. These are treated as free parameters. If UHECR sources have a spectral index steeper than 2 , the total injection energy from a source could exceed the energy budget of possible candidates of UHECR sources. Thus, $E_{\min }$ is required as a cutoff. For conserving energetics, a broken power-law spectrum, for example, $\alpha=2$ at 
lower energy than an energy $E_{0}$, which is a break energy of the spectral index as parameter, was proposed $[40,41] . E_{\min }$ in this study should be interpreted as a minimum energy if UHECR injection spectrum can be expressed by a single spectral index.

Taking these source-evolution models into account, the total neutrino flux from all sources is calculated as

$$
\frac{d N}{d E d t}\left(E_{\nu}\right)=\int_{0}^{5} d z f_{\mathrm{ev}}(z) \int d E_{p} \frac{d n}{d t}\left(E_{\nu}, E_{p}, z\right)
$$

where $f_{\text {ev }}(z)$ and $d n / d t\left(E_{\nu}, E_{p}, z\right)$ are the source-evolution factor and the number of cosmogenic neutrinos with energy $E_{\nu}$ at the Earth produced by protons with energy $E_{p}$ injected from a source at redshift $z$ per time at $z=0$. The normalization factor of the neutrino flux and the value of $\alpha$ are determined by fitting the observed UHECR spectra.

\section{Results}

In this section, the results of our calculations of the fluxes of cosmogenic neutrinos are described. First of all, we compare our calculated spectrum to a spectrum estimated by Ref. [13] to check our simplified treatment of photopion production and to investigate the difference of the neutrino spectra predicted from 2 different IR/UV background models in Section 3.1. In Section 3.2, the normalization factors of the neutrino flux and the spectral indices of UHECR injection at sources are determined for every source-evolution model and transition scenario so that the calculated spectra of UHE protons best reproduce the observed spectra. Then, we investigate the parameter dependence of the neutrino fluxes and discuss the capability to judge which transition scenario is favored in Section 3.3. The detectability of cosmogenic neutrinos is also discussed.

\subsection{Comparison with Another Estimation}

Fig.2 shows a $\nu_{\mu}+\overline{\nu_{\mu}}$ spectrum calculated in this study (red solid line) and that in Ref.[13] (blue solid line). In addition, our spectrum is divided into two spectra: the contributions of the CMB photons (red dashed line) and the others (red dot-dashed line). Differences between the two calculations are the treatment of photopion production and IR/UV background models. Photopion production is treated by a simplified method explained in Section 2 in this 


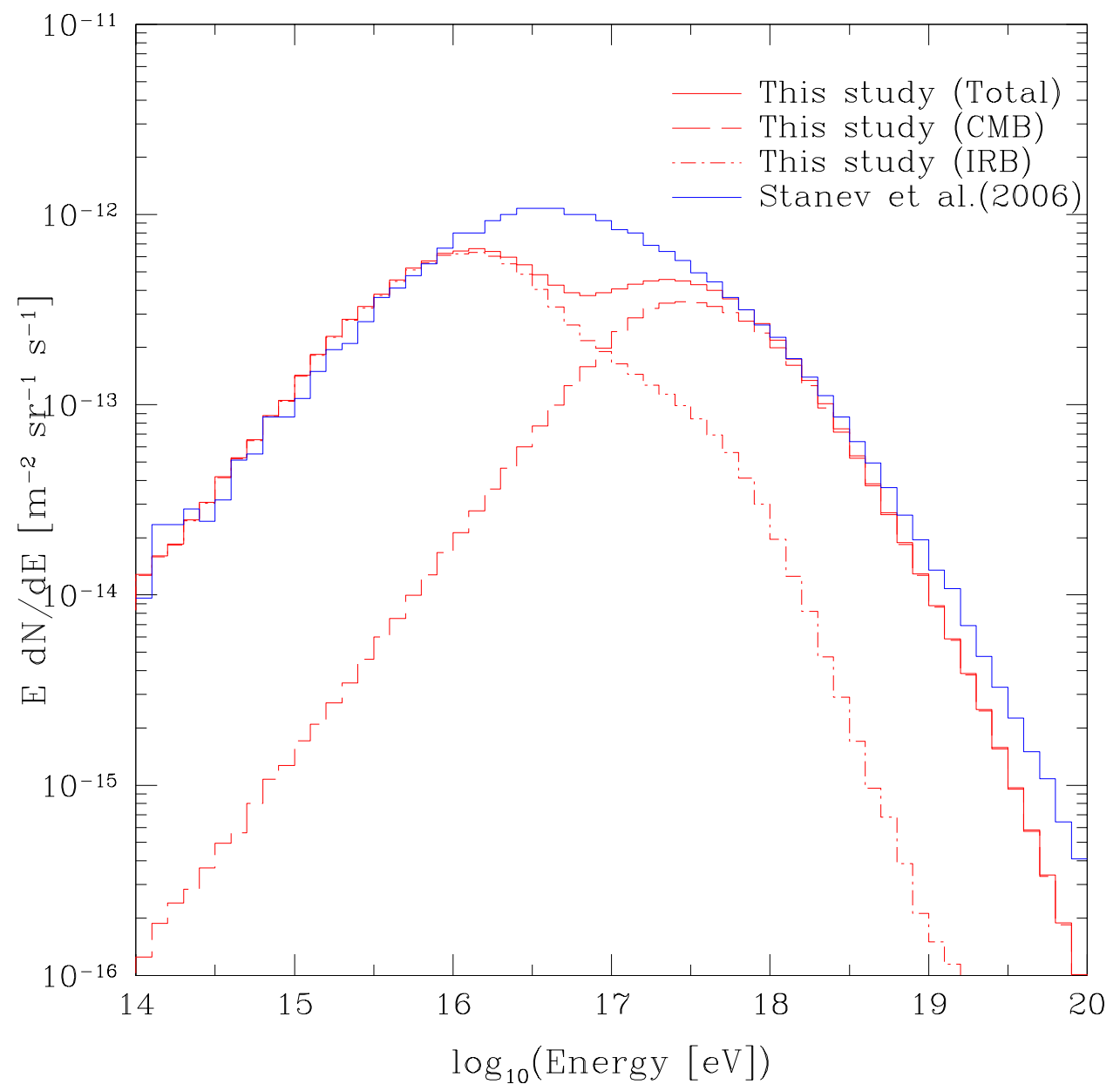

Fig. 2. Comparison of our $\nu_{\mu}+\overline{\nu_{\mu}}$ spectrum (red solid line) to that calculated in Ref.[13] (blue solid line). The same models and parameters are adopted for both calculations except for the treatment of photopion production and IR/UV background models. Our result is divided into contributions by only the CMB (red dashed line) and that by the others (red dot-dashed line). The flux difference at $\sim 10^{17} \mathrm{eV}$ results from different IR/UV background models.

study, while fully simulated by using an event generator SOPHIA [42] in Ref. [13]. For IR/UV background photons, we adopt a model constructed by Ref. [15], whereas Ref. [13] used another model by Ref. [16]. The other parameters are the same ones for comparison: $E_{\max }=10^{21.5} \mathrm{eV}, E_{\min }=10^{18} \mathrm{eV}$, the proton injection spectrum of $d N / d E \propto E^{-2.5} \exp \left(-E / E_{\max }\right) \Theta\left(E-E_{\min }\right)$, and a strong source evolution model defined in Ref.[13]. These fluxes are normalized by the same method as in Ref.[13].

Our calculation well agrees with the estimation of Ref. [13], but the neutrino fluxes at $\sim 10^{17} \mathrm{eV}$ and above $10^{19.5} \mathrm{eV}$ are about a factor of 2 smaller than those by Ref. [13]. 
The discrepancy of the flux at $\sim 10^{17} \mathrm{eV}$ is explained by the difference of adopted IR/UV background models as follows. At $z=0$, the number density of photons in the background model of Ref.[15] is about twice as small as that of Ref.[16] at $\sim 0.2 \mathrm{eV}$. Since photopion production dominantly occurs through the delta resonance, $s \sim 1.6 \mathrm{GeV}^{2}$, the energy of protons which dominantly interact with photons of $0.2 \mathrm{eV}$ is $\sim 2 \times 10^{18} \mathrm{eV}$ and the energy of produced neutrinos is $\sim 10^{17} \mathrm{eV}$. The mean free path of these protons for photopion production is $\sim 10^{5} \mathrm{Mpc}$ at $z=0$ (see Fig. 1), and therefore the Universe is transparent against these protons. Thus, the number of interactions is proportional to the photon number density, and then the IR/UV background radiation model by Ref.[16] leads to the neutrino flux twice as large as that in the background model used in this study at $\sim 10^{17} \mathrm{eV}$. Note that these neutrinos are not generated at high-redshift Universe because the energy-loss length of Bethe-Heitler pair-creation is much shorter than the interaction length of photopion production (see Fig.1). Hence, a discussion above at $z=0$ is sufficient. The shape of the spectrum of cosmogenic neutrinos gives us useful information on IR background photons.

The flux difference above $10^{19.5} \mathrm{eV}$ originates from our simplified treatment of photopion production. Our treatment predicts a little lower flux than in Ref. [13] at the highest energy.

\subsection{Normalization of the Neutrino Flux}

The calculated fluxes of cosmogenic neutrinos are normalized so as to give a good fit to observed UHECR spectra in this study. The spectral fit constrains the spectral indices of the proton injection spectra and the normalization factors of the neutrino fluxes through the normalization of UHECR fluxes at the same time. We adopt UHECR spectra observed by the Akeno $[43,44]$ and AGASA [45], and by the HiRes-I and HiRes-II [46] for the normalization. Note that the AGASA spectrum is shifted by $10 \%$ to lower energies to give a good agreement with the Akeno spectrum [47].

Fig.3 represents calculated proton spectra which are best fitted to the observed spectra for different source-evolution models listed in the figure. In the upper two panels, the calculated spectra are fitted using the chi-square method in the range from $10^{18.0}$ to $10^{19.6} \mathrm{eV}$, which correspond to the proton-dip scenario. As a result, spectral indices which best reproduce the Akeno-AGASA results are $\alpha=2.7,2.5,2.5$ and 2.3 for the UNF, QSO, SFR, and SFR+GRBMAC source-evolution models, respectively. Fitting to the HiRes spectra leads to $\alpha=2.6,2.5,2.5$, and 2.3, respectively. These indices are independent of $E_{\min }$ and $E_{\max }$ as long as $E_{\min }<10^{18} \mathrm{eV}$ and $E_{\max }>10^{20} \mathrm{eV}$. In the lower two panels, we fix the spectral indices to 2.0 and try to fit the calculated spectra 


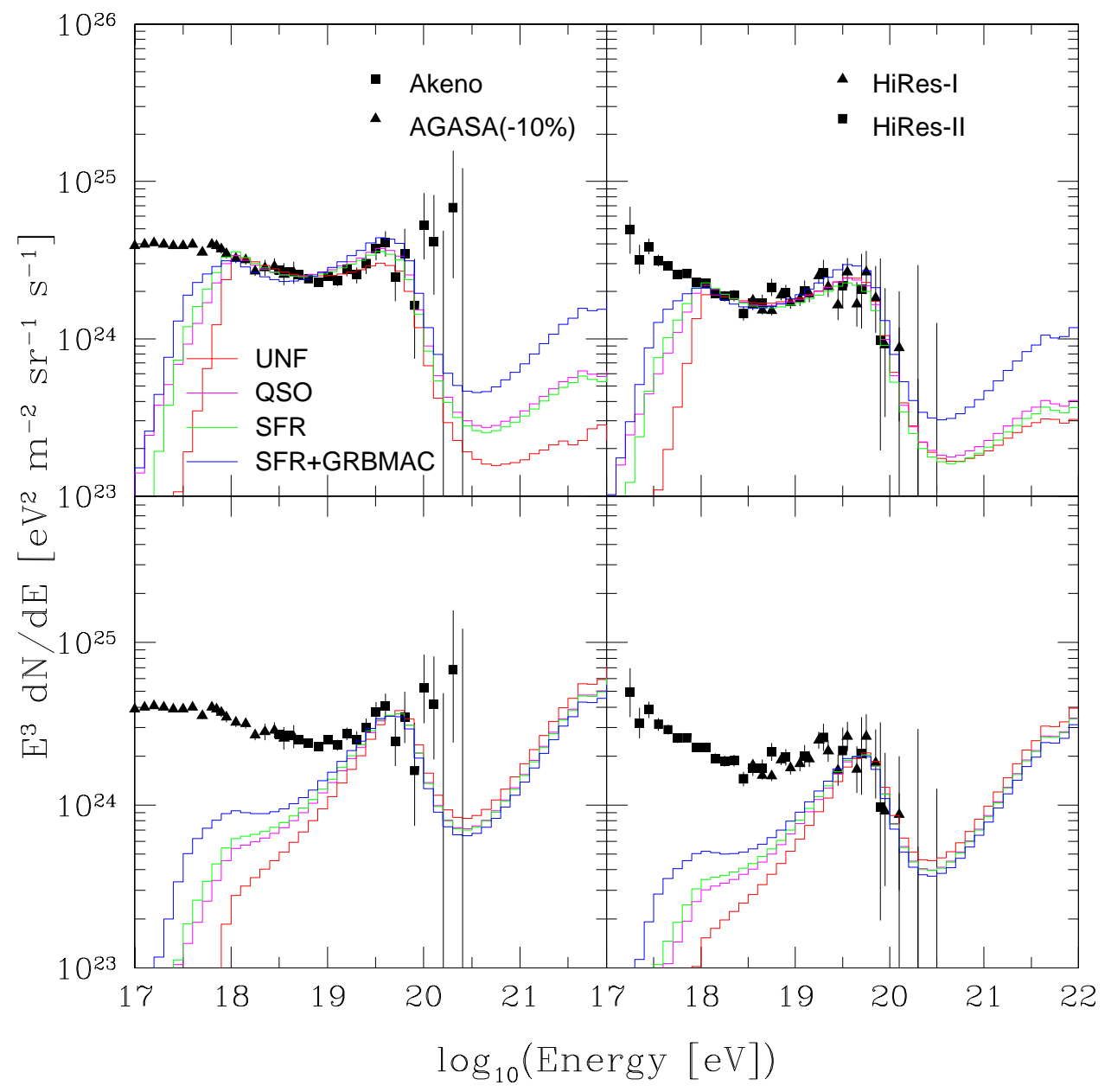

Fig. 3. Proton spectra fitted to the Akeno-AGASA data (left two panels) and HiRes data (right two panels). Note that a spectrum by the AGASA is shifted by $10 \%$ to the lower energy to give a good agreement with the Akeno spectrum. $E_{\max }$ and $E_{\min }$ are set to be $10^{22} \mathrm{eV}$ and $10^{18} \mathrm{eV}$, respectively. The upper two panels correspond to the proton-dip scenario. The spectral indices of proton sources are 2.7, 2.5, 2.5, and 2.3 for UNF, QSO, SFR, and SFR+GRBMAC source-evolution models, respectively in the upper left panel. In the upper right panel, the spectral indices are 2.6, 2.5, 2.5 , and 2.3, respectively. These are the best fit spectral indices of $\chi^{2}$ fittings with the observational spectra from $10^{18.0}$ to $10^{19.6} \mathrm{eV}$. In the lower two panels, which are in the case of the ankle-transition scenario, the spectral indices are set to be 2.0 .

to the observed ones in the energy range from $10^{19.5}$ to $10^{19.9} \mathrm{eV}$ to represent the ankle-transition scenario. In both scenarios, shortfalls in the fluxes at lower energies are thought to be compensated by GCRs. Throughout this paper, we adopt the normalization based on the Akeno-AGASA spectrum.

The PAO also reported the energy spectra of UHECRs [48]. However, an energy spectrum derived from its ground-based detector covers only down to 

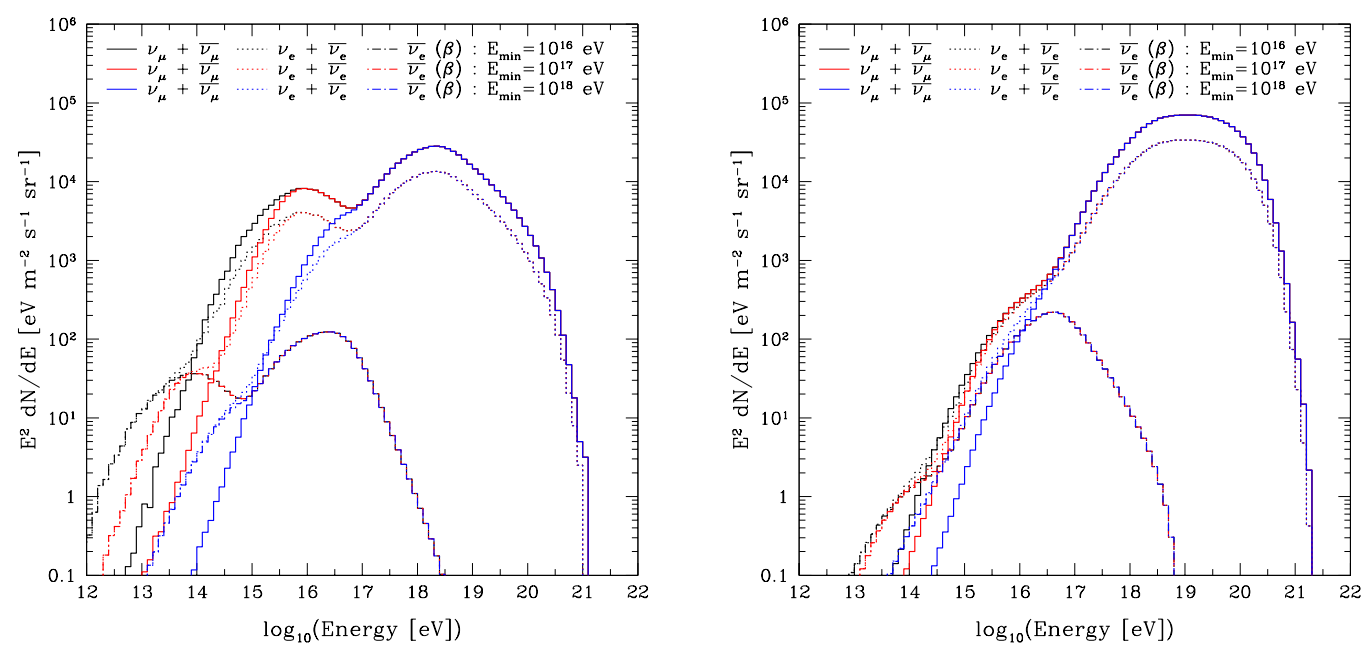

Fig. 4. Spectra of cosmogenic neutrinos with $E_{\min }=10^{16}$ (black) $10^{17}$ (red) and $10^{18} \mathrm{eV}$ (blue) in the proton-dip scenario (left) and the ankle-transition scenario (right). The solid lines, dotted lines, and dot-dashed lines show the spectra of $\nu_{\mu}+\overline{\nu_{\mu}}$, $\nu_{e}+\overline{\nu_{e}}$, and $\overline{\nu_{e}}$ from neutron beta decay, respectively. The cosmological evolution of UHECR sources and neutrino oscillation are not taken into account. All fluxes are normalized by the Akeno-AGASA spectrum.

$10^{18.4} \mathrm{eV}$. An energy spectrum constructed from a hybrid method covers above $10^{18} \mathrm{eV}$, but it has a large statistical error due to the small number of detected events above $10^{19} \mathrm{eV}$. Thus, we do not use the PAO spectra in this study.

\subsection{Neutrino Fluxes}

We start by investigating the dependence of the fluxes of cosmogenic neutrinos on $E_{\text {min }}$. Fig.4 shows the calculated spectra of $\nu_{\mu}+\overline{\nu_{\mu}}$ (solid lines), $\nu_{e}+\overline{\nu_{e}}$ (dotted lines), $\overline{\nu_{e}}$ from neutron beta decay (dot-dashed lines) for $E_{\min }=10^{16}$ (black), $10^{17}$ (red), and $10^{18} \mathrm{eV}$ (blue). These spectra are calculated based on the proton-dip (left) and ankle-transition (right) scenarios. The cosmological evolution of UHECR sources and neutrino oscillation are not taken into account. The $\nu_{e}+\overline{\nu_{e}}$ fluxes are twice as low as the $\nu_{\mu}+\overline{\nu_{\mu}}$ fluxes in the energy range where neutron beta decay does not contribute to $\overline{\nu_{e}}$ fluxes because pion decay produces two muon neutrinos and one electron neutrino.

An intriguing feature is the spectral peaks of both $\nu_{\mu}+\overline{\nu_{\mu}}$ and $\nu_{e}+\overline{\nu_{e}}$ spectra at $\sim 10^{16} \mathrm{eV}$ in the left panel. These peaks are generated by interactions between protons with $\sim 10^{17} \mathrm{eV}$ and UV photons. Since there are many protons at lower energies due to a steep injection spectrum, UV background photons significantly contribute to the neutrino flux. On the other hand, we cannot see a spectral peak at $\sim 10^{16}$ in the right panel. The spectral peaks are a characteristic feature in the proton-dip scenario. 
We should take neutrino oscillation into account for discussions of the detectability of cosmogenic neutrinos, as we can only observe the spectrum of neutrinos which suffers from neutrino oscillation during their propagation. Neutrinos generated from charged pions and successive muon decay have a flavor ratio of $\nu_{e}: \nu_{\mu}: \nu_{\tau}=1: 2: 0$. Neutrino oscillation changes this ratio into $\sim 1: 1: 1$ [49]. The flavor ratio approximately holds $1: 1: 1$ even at low energies unless anti-neutrinos from neutron $\beta$ decay are dominated.

Fig. 5 shows the spectra of cosmogenic neutrinos per flavor, $\nu_{i}+\overline{\nu_{i}}$, taking neutrino oscillation into account. $E_{\min }$ and $E_{\max }$ are set to $10^{16}$ and $10^{22} \mathrm{eV}$, respectively. Upper limits of neutrino fluxes determined by several experiments and the sensitivities of current/future neutrino detectors are also displayed. Theoretically estimated fluxes of diffuse neutrinos from AGNs [58] and GRBs [59] are also shown.

As mentioned above, the proton-dip scenario predicts a spectral peak at $\sim 10^{16}$ $\mathrm{eV}$, while the peak does not appear for the ankle-transition scenario. The flux difference at $10^{16} \mathrm{eV}$ between the two scenarios is about an order of magnitude. Therefore, the detection of this peak could be an evidence of the proton-dip scenario. The flux of the peak depends on source-evolution models. The SFR and SFR+GRBMAC source-evolution models result in three and four times higher neutrino fluxes than the UNF source-evolution model, respectively. In these strong source-evolution models, the predicted fluxes are comparable with the IceCube 3yr sensitivity.

The neutrino flux at $\sim 10^{20} \mathrm{eV}$ could be also a clue to judge a better transition scenario. The ankle-transition scenario leads to the neutrino flux much higher than the proton-dip scenario at the highest energy because of a harder injection spectrum. In the SFR source-evolution model, the estimated flux reaches the full-ANITA sensitivity. Remember that our estimation of the flux of cosmogenic neutrinos above $10^{19.5} \mathrm{eV}$ might be underestimated by about a factor of 2 as shown in Section 3.1. This works positively for the detection of the highest energy neutrinos. The full-ANITA is also expected to detect the highest energy neutrinos given the SFR+GRBMAC model is realistic enough, and it implies that GRBs are UHECR sources, though we should keep in mind that the models of the GRB rate history include large uncertainty 2 .

The neutrino flux at the highest energy strongly depends on $E_{\max }$. Fig.6 shows the spectra of cosmogenic neutrinos calculated for $E_{\max }=10^{22.0}, 10^{21.5}$, and $10^{21.0} \mathrm{eV}$ in the SFR source-evolution model. All of $E_{\max }$ can reproduce the observed cosmic ray spectra sufficiently. We can see that the neutrino fluxes above $10^{19} \mathrm{eV}$ are sensitive to $E_{\max }$. As $E_{\max }$ becomes smaller, the neutrino

$\overline{2}$ A preliminary upper bound of the ANITA approaches the predicted flux of cosmogenic neutrinos with $E_{\max }=10^{22} \mathrm{eV}$ in the ankle-transition scenario at $\sim 10^{20}$ $\mathrm{eV}$ (see http://www.slac.stanford.edu/econf/C070730/). 


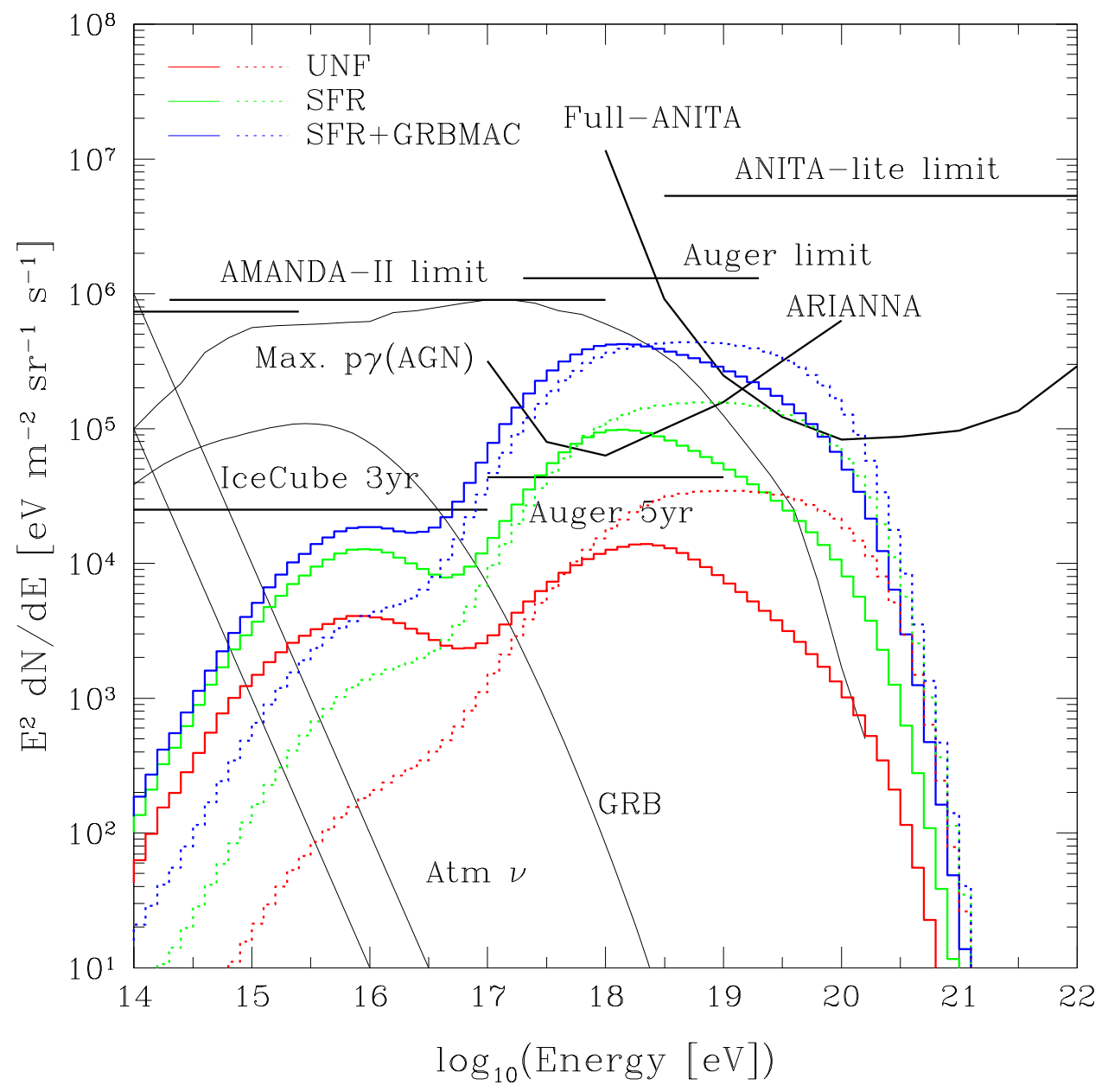

Fig. 5. Predicted spectra of cosmogenic neutrinos per flavor $\left(\nu_{i}+\bar{\nu}_{i}\right)$ in the proton-dip scenario (solid lines) and ankle-transition scenario (dotted lines). These fluxes are normalized by using the Akeno-AGASA spectrum. $E_{\min }$ and $E_{\max }$ are set to $10^{16}$ and $10^{22} \mathrm{eV}$. The red lines, green lines, and blue lines are neutrino spectra for the UNF, SFR, and SFR+GRBMAC source-evolution models, respectively. The fluxes of the atmospheric neutrinos (Atm $\nu$ vertical/horizontal) [50] are represented. As upper limits of neutrino fluxes by several experiments, AMANDA-II limits [51,52], limit on tau neutrinos by the PAO [53], ANITA-lite limit [54] are shown. As estimated or projected sensitivities, sensitivity of 3 years observation by IceCube [55], sensitivity of 5 years observation by the PAO [56], ARIANNA sensitivity [57], and full-ANITA sensitivity [54] are also shown. As diffuse neutrino spectra from energetic sources, a maximal neutrino flux from active galactic nuclei including neutrino oscillation [58] and neutrino spectrum from GRBs calculated in Ref.[59], considering neutrino oscillation, with their parameters of $E_{\text {jet }}=1.24 \times 10^{51} \mathrm{erg}, E_{\mathrm{sh}}=10^{51} \mathrm{erg}, \xi_{\mathrm{B}}=1$, $\xi_{\text {acc }}=100, \Gamma=10^{2.5}, r=10^{13}-10^{14.5} \mathrm{~cm}$ and $l=r / \Gamma=10^{10.5} \mathrm{~cm}$, which are used in Ref.[60], are shown.

flux at the highest energy is smaller and the flux difference between the two scenarios also becomes smaller. In order that the neutrino flux at $\sim 10^{20} \mathrm{eV}$ 
can be a clue to distinguish between the two scenarios, sufficiently large $E_{\text {max }}$ is required.

Whereas the neutrino fluxes at $\sim 10^{18} \mathrm{eV}$ are independent of not only the transition scenarios (see Fig.5) but also $E_{\min }$ and $E_{\max }$ (see Figs.4 and 6), the neutrino fluxes are sensitive to source-evolution models. Thus, the neutrino flux at $\sim 10^{18} \mathrm{eV}$ has information on the cosmological evolution of UHECR sources. The ARIANNA and PAO will detect cosmogenic neutrinos as long as the SFR or SFR+GRBMAC source-evolution scenarios are good ones. We can also obtain indirect knowledge on UHECR sources by comparing sourceevolution models constrained by the neutrino observatories to the cosmological evolution models of various astrophysical objects.

The spectral features of cosmogenic neutrinos described above might be covered by the neutrino background from powerful objects like GRBs and AGNs, as shown in Fig.5. If the neutrino fluxes from these energetic objects are larger than the flux of cosmogenic neutrinos, it would be difficult to test transition scenarios by cosmogenic neutrinos.

A diffuse neutrino flux from GRBs estimated by Ref. [59] is larger than the predicted fluxes of cosmogenic neutrinos up to $\sim 10^{17} \mathrm{eV}$ and hides the spectral peaks of cosmogenic neutrinos at $\sim 10^{16} \mathrm{eV}$ which is predicted in the proton-dip scenario. However, since neutrinos from GRBs are, in principle, distinguishable from cosmogenic neutrinos by time and spatial correlations between prompt $\gamma$-rays and neutrinos, the spectral peak can be detected.

For a diffuse neutrino flux from AGNs, the prediction of a maximal contribution model by Ref. [58] is shown in Fig. 5. The estimation covers not only the peak of the spectrum of cosmogenic neutrinos at $\sim 10^{16} \mathrm{eV}$ for the protondip scenario but also the neutrino flux at $\sim 10^{18} \mathrm{eV}$. Unfortunately, spatial correlation between emitted neutrinos and photons is not expected for distant AGNs, because AGNs are not as bright as GRBs in general. However, we should notice that this flux is estimated as a maximum. The total flux of diffuse neutrinos starts to be restricted by AMANDA-II [52] and will be constrained more precisely by observations in the near future by detectors such as IceCube. Whether the spectral features of cosmogenic neutrinos are not covered by AGN diffuse neutrinos and are observable will be determined by observations.

\section{Discussion}

In Section 3, we found that the spectrum of cosmogenic neutrinos can be an indicator to distinguish the two GCR/EGCR transition scenarios though its 


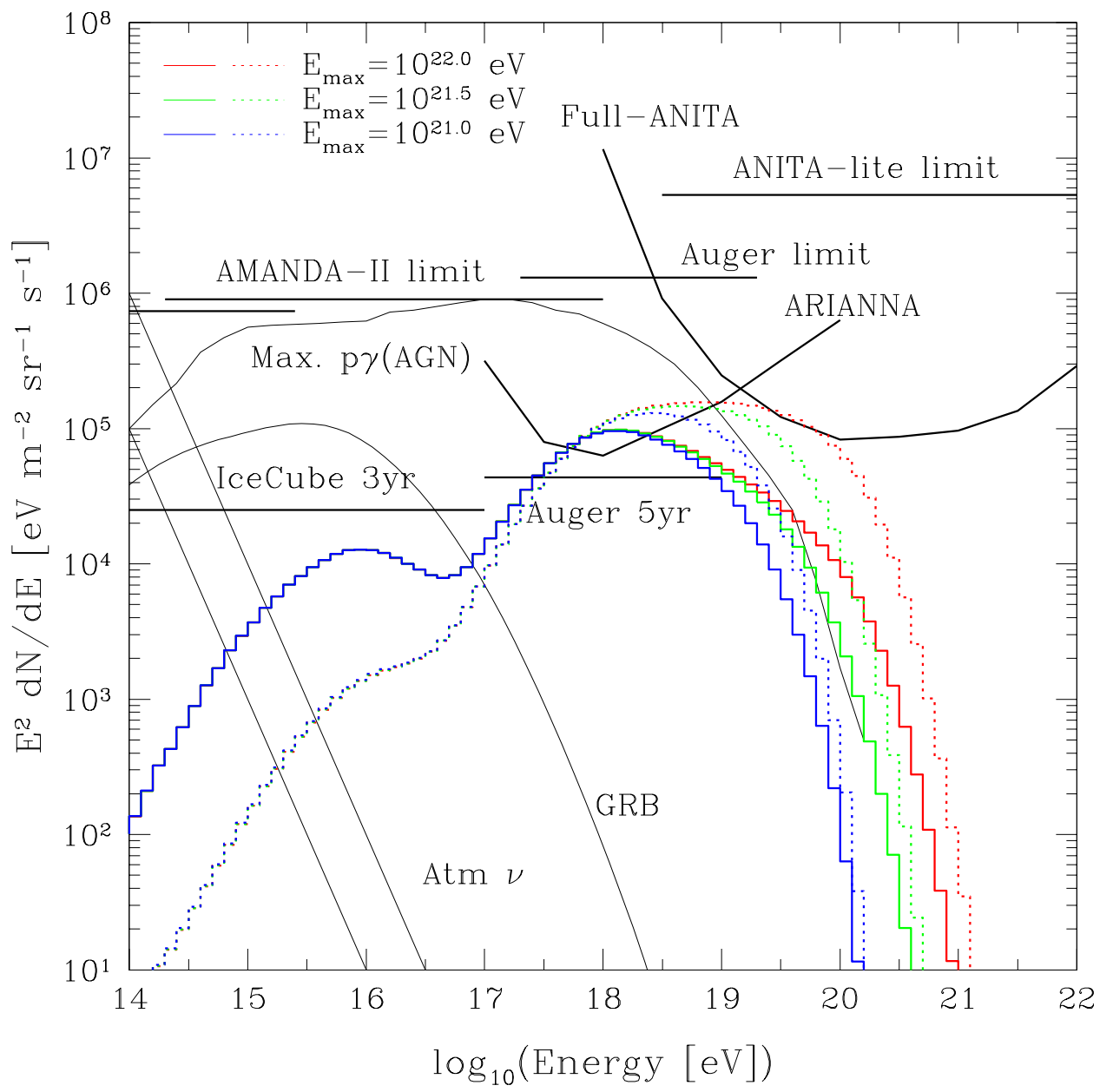

Fig. 6. The same as Fig.5, but spectra only calculated for the SFR source-evolution model in the cases of different $E_{\max }$, set to $10^{22}$ (red lines), $10^{21.5}$ (green lines), and $10^{21} \mathrm{eV}$ (blues lines). The solid lines are spectra based on the proton-dip scenario, and the dotted lines are those on the ankle-transition scenario.

capability depends on several parameters on UHECR sources: $E_{\min }, E_{\max }$, and cosmological evolution models of UHECR sources. In this section, we discuss other uncertainties on the flux of cosmogenic neutrinos and their detectability.

The difference between the Akeno-AGASA and HiRes spectra brings the uncertainty of the neutrino flux through the normalization of the UHECR flux. The UHECR flux observed by the HiRes is twice as low as the Akeno-AGASA as shown in Fig.3 as long as the systematic errors of the determination of UHECR energies of both experiments are neglected. The flux difference is reflected to the flux of cosmogenic neutrinos directly, and therefore the difference of the neutrinos fluxes between the two normalizations is about a factor of 2 .

A calibration of the energy-scale of UHECR observations could decrease the uncertainty of the neutrino flux. A dip calibration method in the proton- 
dip scenario $[2,3]$ leads to a good agreement between the Akeno-AGASA and HiRes spectra assuming that the HiRes spectra are shifted to higher energy by a factor of 1.2 and the AGASA spectrum is shifted to lower energy by a factor of 0.9. Since the latter shift has already been performed for giving a good agreement with the Akeno spectrum, the normalization of the neutrino flux based on the shifted HiRes spectra results in the neutrino flux comparable with the Akeno-AGASA normalization if the proton-dip scenario is true.

Since we have not know which transition scenario is favored yet, the uncertainty on the neutrino flux which originates from the difference of observed UHECR fluxes is maximally a factor of 2 .

The number density of IR/UV background photons also causes uncertainty on the flux of cosmogenic neutrinos. We already discussed the difference of the neutrino fluxes between different IR/UV background models of Refs. [15,16]. Since the number density of infrared photons in the model of Ref. [16] is twice as large as that of Ref. [15] at low redshift, the former model predicts the neutrino flux twice as large as the latter model at $\sim 10^{17} \mathrm{eV}$. Here, we also compare the best-fit model, which is adopted in this study, with a low-infrared model in the same reference. At $z=0$, the number density of photons in the range of $0.005-0.5 \mathrm{eV}$ in the best-fit model is about twice as large as that in the low-infrared model. This energy range of photons corresponds to the neutrino energy of $4 \times 10^{16}-4 \times 10^{18} \mathrm{eV}$. Thus, a neutrino flux predicted in the low-infrared model is twice as small as that in the best-fit model in this energy range. In fact, the CMB photons mainly contribute to the total neutrino flux above a few $\times 10^{17} \mathrm{eV}$. The low-infrared model predicts the neutrino flux twice as small as the best-fit model at $\sim 10^{16}-10^{17} \mathrm{eV}$. Adding the result of the discussion in Section 3.1 to the discussion above, the uncertainty of the neutrino flux at $\sim 10^{16}-10^{17} \mathrm{eV}$ is a factor of 4 .

The difference of the spectral index of UHECR injection also slightly changes the neutrino flux. The spectral index has been fixed to 2.0 for the ankletransition scenario in Section 3, but somewhat steeper injection spectrum is also allowed as mentioned in Section 1. We check the variation of the neutrino flux resulting from the change of the spectral index. Fig. 7 shows the comparisons of the neutrino fluxes predicted from two spectral indices in the ankle-transition scenario. For reference, a predicted spectrum in the protondip scenario is also shown. The flux difference between the two transition scenarios becomes smaller for a steeper spectrum in the ankle-transition scenario, which is about a factor of 3 at $\sim 10^{16}$ and $10^{20} \mathrm{eV}$. In that case, the distinction between the two scenarios becomes little clear.

The composition of UHECRs could affect the flux of cosmogenic neutrinos. The composition is poorly known as mentioned in Section 1. Here, we consider only a mixed composition model adopted in Ref. [14]. This model is an 


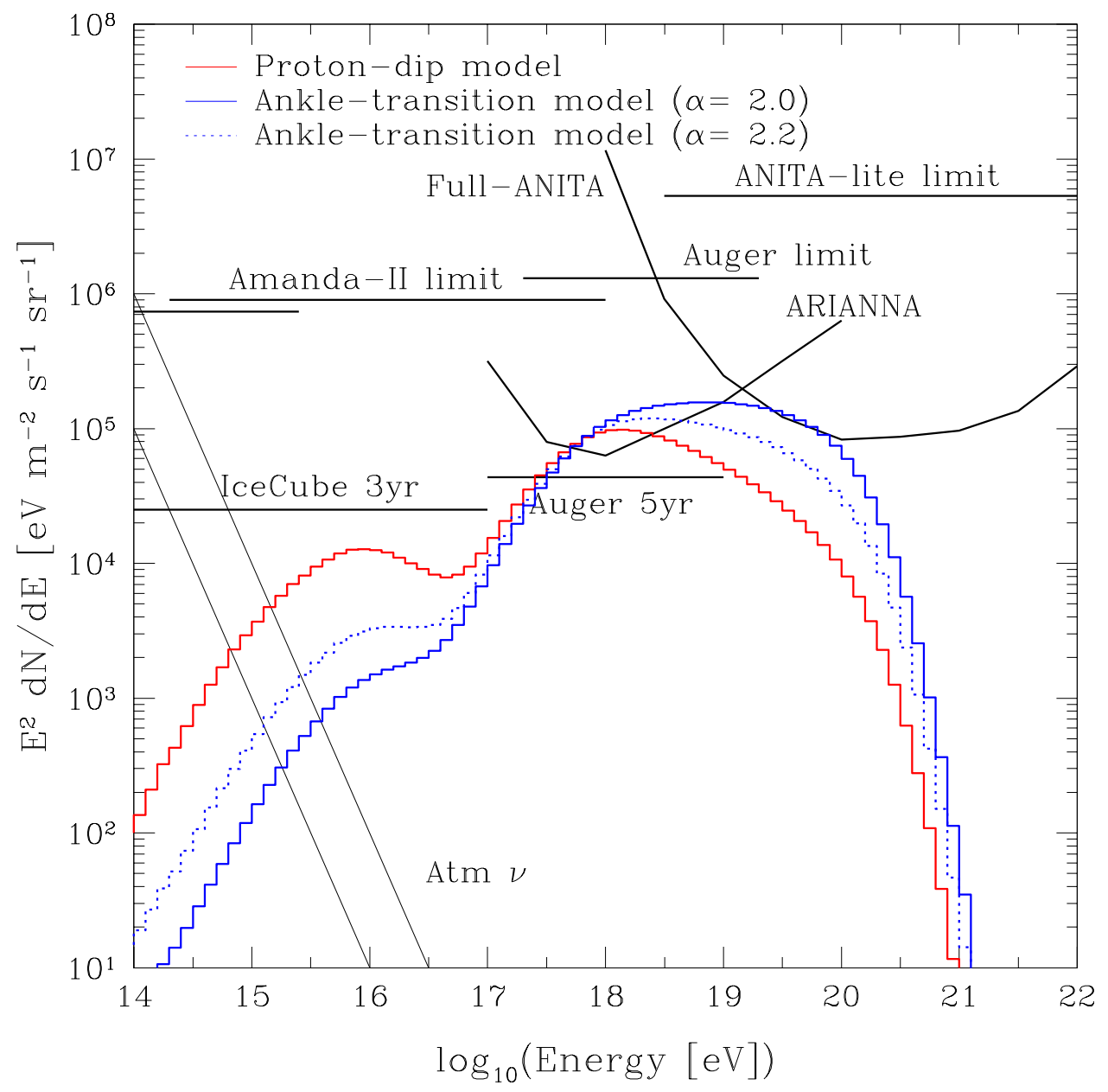

Fig. 7. Comparison between cosmogenic neutrino spectra with different spectral indices in the ankle-transition scenario. The blue solid line and blue dotted line are neutrino spectra with $\alpha=2.0$ and 2.2, respectively. The red line is neutrino spectrum in the proton-dip scenario for reference. These spectra are calculated in the SFR source-evolution model with $E_{\max }=10^{22} \mathrm{eV}$ and $E_{\min }=10^{16} \mathrm{eV}$. Solid black lines are the same as those in Fig.5

improved version of the ankle-transition scenario. Fig.9 of Ref.[14] shows that nuclei heavier than protons contribute to the neutrino spectrum mainly below $10^{15} \mathrm{eV}$. Since the neutrinos below $10^{15} \mathrm{eV}$ are covered by atmospheric neutrinos as shown in Fig.5 and 6, protons make a dominant contribution to the flux of cosmogenic neutrinos in an observable energy range in the ankle-transition scenario. Thus, the capability to distinguish the transition scenarios is not dramatically affected.

Finally, we revisit diffuse neutrino fluxes from AGNs and GRBs and discuss the detectability of cosmogenic neutrinos. In Fig.5, the diffuse neutrino flux from AGNs, maximally estimated in Ref.[58], is shown. This estimation assumes that $\mathrm{GeV}-\mathrm{TeV} \gamma$-rays from AGNs are of hadronic origin. This scenario requires 
high-energy hadrons which can generate neutrinos with energies comparable to cosmogenic neutrinos. Therefore, a large flux of high-energy neutrinos is predicted. On the other hand, the $\gamma$-rays can also be reproduced by leptonic origin (for review see [61]). This scenario does not always predict UHE neutrinos because UHECR generation is not required. It is still controversial which model is the favorable one. Thus, whether the features of cosmogenic neutrino spectrum can be observed or not is related to the origin of high-energy $\gamma$-rays.

GRBs are also possible candidates of the sources of UHE neutrinos. In principle, high-energy neutrinos from GRBs can be distinguished from cosmogenic neutrinos since such neutrinos correlate with the prompt $\gamma$-rays of GRBs. However, recently, it has been suggested that neutrinos from low-luminosity GRBs can contribute to high-energy diffuse neutrino background [62]. Such neutrinos might be difficult to distinguish since the prompt emissions from such GRBs are difficult to observe. That neutrino flux can be comparable to the flux of cosmogenic neutrinos, $\sim 10^{4} \mathrm{eVm}^{-2} \mathrm{sr}^{-1} \mathrm{~s}^{-1}$ at $\sim 10^{16} \mathrm{eV}$, but it strongly depends on the local rate of the low-luminosity GRBs, which has large uncertainties.

As discussed above, diffuse neutrinos from several powerful objects could prevent us from observing the spectral features of cosmogenic neutrinos. It is determined by future observations whether neutrinos from these objects can hide the spectral features.

\section{Conclusion}

We calculated the fluxes of cosmogenic neutrinos for several plausible parameter sets and discussed the possibility that the neutrino flux helps to judge which scenario of transition from GCRs to EGCRs is favored. We found that the spectrum of cosmogenic neutrinos has a characteristic peak at $\sim 10^{16} \mathrm{eV}$ in the proton-dip scenario as long as extragalactic protons significantly contribute to the observed cosmic rays down to $10^{17} \mathrm{eV}$. The predicted flux is comparable with the sensitivity of IceCube when the SFR source-evolution model is considered. On the other hand, we also found that the neutrino flux at $\sim 10^{20} \mathrm{eV}$ is much larger in the ankle-transition scenario than in the proton-dip scenario if the maximum energy of protons generated at sources is sufficiently high. If the SFR+GRBMAC source-evolution model is appropriate one, neutrinos with $\sim 10^{20} \mathrm{eV}$ are expected to be detected by Full-ANITA though the flux is highly dependent on $E_{\max }$. These spectral features give us clues to judge which scenario is favored unless these are covered by the neutrino background from potential neutrino sources like AGNs and GRBs.

We also found that the neutrino flux at $\sim 10^{18} \mathrm{eV}$ depends on only the cos- 
mological evolution of UHECR sources. This indicates that the neutrino flux at this energy brings us information on the cosmological evolution of UHECR sources. The detection of this feature is feasible by ARIANNA and PAO if UHECR sources cosmologically evolve like star formation rate.

As discussed in this paper, the spectrum of cosmogenic neutrinos depends on many unknown parameters: $E_{\max }, E_{\min }$, source-evolution models, scenarios of the GCR/EGCR transition, the shape of the SED of IR/UV background photons. That is why cosmogenic neutrinos are the messengers of not only the nature of the EGCR sources but also cosmic background radiation. The future detection of cosmogenic neutrinos will provide us a lot of information on the Universe related to the highest energy phenomena.

\section{Acknowledgements:}

We thank Tanja Kneiske for publishing the tables of cosmological evolution of the infrared background from Ref.[15] at her website. We are grateful to Motohiko Nagano, Masahiro, Teshima, and Masaki Fukushima for providing Akeno data and useful comments. We are thankful to Shunsaku Horiuchi for helpful comments. The works of H.T. and K.M. are supported by Grants-in-Aid for JSPS Fellows. This work is partially supported by Grants-in-Aid for Scientific Research from the Ministry of Education, Culture, Sports, Science and Technology of Japan through No.19104006 (K.S. and S.N.), No.19047004, and No.19740139 (S.N.), Grant-in-Aid for the 21st Century COE 'Center for the Diversity and Universality in Physics' from the Ministry of Education, Culture, Sports, Science and Technology (MEXT) of Japan, and World Premier International Research Center Initiative (WPI Initiative), MEXT, Japan.

\section{References}

[1] T. Stanev, High Energy Cosmic Rays, Springer-Varlag, 2004, pp.11

[2] V. Berezinsky, A. Gazizov, S. Grigorieva, Phys. Lett. B612 (2005) 147

[3] R. Aloisio et al., Astropart. Phys. 27 (2007) 76

[4] R.U. Abbasi, Astrophys. J. 622 (2005) 910

[5] M. Unger et al., arXiv:0706.1495

[6] V. Berezinsky, G. Zatsepin, Phys. Lett. B 28 (1969) 423

[7] F.W. Stecker, Astrophys. Space Sci. 20 (1973) 47

[8] F.W. Stecker, Astrophys. J. 228 (1979) 919 
[9] S. Yoshida, M. Teshima, Prog. Theor. Phys. 89 (1993) 833

[10] R. Engel, D. Seckel, T. Stanev, Phys. Rev. D 64 (2001) 093010

[11] D. Seckel, T. Stanev, Phys. Rev. Lett. 95 (2005) 141101

[12] M. Ave et al., Astropart. Phys. 23 (2005) 19

[13] M. de Marco et al., Phys. Rev. D 73 (2006) 043003

[14] D. Allard et al., JCAP 09 (2006) 005

[15] T.M. Kneiske et al., Astron. Astrophys. 413 (2004) 807

[16] F.W. Stecker, M.A. Malkan, S.T. Scully, Astrophys. J. 648 (2006) 774

[17] T. Stanev, astro-ph/0611633

[18] R. Engel et al., arXiv:0706.1921

[19] A.V. Glushkov et al., JETP Lett. 87 (2008) 190

[20] The Pierre Auger Collaboration, Science 318 (2007) 938

[21] The Pierre Auger Collaboration, Astropart. Phys. 29 (2008) 188

[22] V.S. Berezinsky, S.I. Grigorieva, Astron. Astrophys. 199 (1988) 1

[23] R.J. Protheroe, P.A.Johnson, Astropart. Phys. 4 (1996) 253

[24] S. Agostinelli et al., 2003 Nucl. Instrum. Methods Phys. Res. A 506 (2003) 250, http://wwwasd.web.cern.ch/wwwasd/geant//geant4.html

[25] K. Greisen, Phys. Rev. Lett. 16 (1966) 748

[26] G.T. Zatsepin, V.A. Kuz'min V A, JETP Lett. 4 (1966) 78

[27] T. Stanev et al., Phys. Rev. D 62 (2000) 093005

[28] M.J. Chodorowski, A.A. Zdziarske, M. Sikora, Astrophys. J. 400 (1992) 181

[29] K. Murase S. Nagataki, Phys. Rev. Lett. 97 (2006) 051101

[30] S. Schadmand, Eur. Phys. J. A 18 (2003) 405

[31] K. Asano, S. Nagataki, Astrophys. J. Lett. 640 (2006) 9

[32] E. Waxman, J. Bahcall, Phys.Rev. D 59 (1999) 023002

[33] E. Waxman, Phys. Rev. Lett. 75 (1995) 386

[34] D. Guetta, T. Piran, JCAP 07 (2007) 003

[35] R. Jimenez, Z. Haiman, Nature 440 (2006) 501

[36] K.Z. Stanek et al., Acta Astron. 56 (2006) 333

[37] A.S. Fruchter et al., Nature 441 (2006) 463 
[38] H. Yüksel, M.D. Kistler, Phys. Rev. D 75 (2007) 083004

[39] S.C. Yoon, N. Langer, C. Norman, Astron. Astrophysics 460 (2006) 199

[40] V. Berezinsky et al., astro-ph/0204357

[41] V. Berezinsky et al., Phys. Rev. D 74 (2006) 043005

[42] A. Mucke et al., Comput. Phys. Commun. 124 (2000) 290

[43] M. Nagano et al., J. Phys. G.: Nucl. Part. Phys. 18 (1992) 423

[44] M. Nagano, 1995 private communication

[45] M. Takeda et al., Astropart. Phys. 19 (2003) 447

[46] R.U. Abbasi et al., Phys. Rev. Lett. 100 (2008) 101101

[47] M. Nagano, A.A. Watson, Rev. Mod. Phys. 72 (2000) 689

[48] T. Yamamoto et al., arXiv:0707.2638

[49] J.G. Learned, S. Pakvasa, Astropart. Phys. 3 (1995) 267

[50] P. Lipari, Astropart. Phys. 1 (1993) 195

[51] A. Achterberg et al., Phys. Rev. D 76 (2007) 042008

[52] M. Ackermann et al., Astrophys. J. 675 (2008) 1014

[53] The Pierre Auger Collaboration, Phys. Rev. Lett. 100 (2008) 211101

[54] S.W. Barwick et al., Phys. Rev. Lett. 96 (2006) 171101

[55] IceCube http://www.icecube.wisc.edu/

[56] X. Bertou et al., Astropart. Phys. 17 (2006) 183

[57] S.W. Barwick, astro-ph/0610631

[58] K. Mannheim, R.J. Protheroe, J.P. Rachen, Phys. Rev. D 63 (2000) 023003

[59] K. Murase, S. Nagataki, Phys. Rev. D 73 (2006) 063002

[60] A. Achterberg et al., Astrophys. J. 664 (2007) 397

[61] F.A. Aharonian, Very High Energy Cosmic Gamma Radiation, World Scientific, 2004, pp.402

[62] K. Murase et al., Astrophys. J. Lett. 651 (2006) 5 